\title{
«QUI A MALES LANTERNES, SOVENT SE TRABUCHE EN VOIE». ALGUNAS DIFICULTADES EN LA TRADUCCIÓN DEL FRANCÉS MEDIEVAL* Berta Pico
}

Universidad de La Laguna

\begin{abstract}
This article deals with certain difficulties in the comprehension of medieval lexicon focusing on aids to support the competence of the language of the period, especially the consultation of dictionaries and the context, as well as the analysis of the manuscripts when possible with regard to the uncritical reception of their editions. To illustrate this, this article shall be assisted by the examples of two texts, both of which are of the medieval period - one of the Old French and the other of the Middle French.
\end{abstract}

KEYWORDS: translation problems, medieval lexicon, old French, middle French.

\section{RESUMEN}

Este artículo trata a algunas dificultades para la comprensión del léxico medieval, atendiendo sobre todo a los medios auxiliares para apoyar la competencia de la lengua de la época, especialmente la consulta de los diccionarios y el contexto, así como el examen de los manuscritos, cuando es posible, frente a la recepción acrítica de sus ediciones. Con el fin de ilustrar el presente artículo, nos serviremos de los ejemplos de dos textos pertenecientes ambos al período medieval, uno al llamado «francés antiguo»y otro al «francés medio».

PALABRAS CLAVE: dificultades de traducción, léxico medieval, francés antiguo, francés medio.

La frase, con aspecto de paremia popular, con la que empieza el título de mi intervención, pertenece al primer apéndice que añadió Jean d'Antioche a su traducción francesa de la obra De inventione de Cicerón y de la anónima 
Rhetorica ad Herennium, acabada en $1282^{1}$. Por entonces, cuando en ese siglo XIII se produjo en Francia un gran movimiento de traducción de los grandes textos latinos técnicos, enciclopédicos o morales, las auctoritates, no era infrecuente que las traducciones fuesen acompañadas de prefacios o apéndices ${ }^{2}$, que constituyen una documentación rica en reflexiones, donde el traductor exponía las dificultades y los problemas lingüísticos que le había planteado la traducción, las divergencias entre las lenguas y la complejidad de sus relaciones, poniendo de manifiesto la exigencia de que el traductor poseyese una gran competencia en las dos lenguas, y al respecto Jean d'Antioche termina su exposición metodológica sobre la traducción advirtiendo a los posibles críticos de su trabajo de que, antes de achacarle errores, cotejen cuidadosamente los dos textos, el latino y el francés, y vean si verdaderamente pueden mejorar su trabajo, pero que tengan cuidado, porque «qui a males lanternes sovent se trabuche en voie », el que lleva malas linternas se cae con frecuencia en el camino.

La advertencia de Jean d'Antioche es válida en cualquier momento, pero muy especialmente en lo que se refiere a la traducción de lenguas, o de estados de lengua, del pasado, para los que el traductor sólo posee competencia de reconocimiento, que, naturalmente, adquiere tras largo tiempo de estudio y de familiaridad con los textos del período, pero, aun así, no está libre de tropiezos y ha de recurrir a distintos medios auxiliares que iluminen los pasajes oscuros. Sin tener en absoluto la pretensión de ser portadora de la luz, sólo pretendo, por el contrario, dar cuenta de algunas dificultades que plantea la traducción del francés medieval, como aclara la segunda parte del título de mi intervención, y proponer la forma de superarlas.

Al reflexionar sobre la traslado de textos literarios medievales a la lengua actual nos viene a la mente la persistencia de la característica más definitoria de las obras medievales, su mouvance, en término de Zumthor, o, si acogemos el de Cerquiglini, su variance, esto es, su movilidad, su inestabilidad, su propagación en el tiempo y en el espacio — nada más alejado del texto medieval que la idea de opus ne varietur-, el continuo remaniement como principio esencial de esta escritura y de la transmisión de la obra, el texto como diasistema, según estableció Segre $^{3}$, como compromiso entre su sistema y el de

\footnotetext{
${ }^{1}$ Jean d'Antioche, ligado a la Orden del Hospital de San Juan de Jerusalén, realizó este trabajo en San Juan de Acre, probablemente a petición de Guillaume de Saint-Étienne, que pocos años más tarde fue comendador de la Orden en Chipre. La traducción, que, bajo el título de Rettorique y bajo el nombre de Cicerón, comprende De inventione y Rhetorica ad Herennium acompañadas de un prólogo y dos apéndices, se conserva en un único manuscrito (Chantilly, Musée Condé, 433).

2 Algunos de ellos han sido editados en la compilación de textos medievales sobre teoría literaria por U. MÖLK (1969), Franæösische Literarästhetik des 12. und 13. Jabrbunderts. Prologe-Exkurse-Epiloge, Tubinga.

${ }^{3}$ C. Segre (1985), Principios de análisis del texto literario, Barcelona, Crítica, p. 383.
} 
cada copista, que no era un mero dibujante de palabras, sino que intentaba comprender lo que leía y hacer comprender lo que escribía, lo que le podía llevar a intervenir decididamente en el texto, pues, como dijo Pierre-Yves Badel, «en chaque copiste il y a un écrivain, un remanieur qui sommeille» ${ }^{4}$. Pero, por tentador que pudiera ser para el traductor actual sentirse un prolongador del copista-escritor medieval, su libertad para intervenir es menor, y la tarea de realizar una buena traducción, en su doble vertiente de comprender y hacer comprender, ya es suficientemente ardua y creativa.

Aunque hoy se asiste a la evasión de la dimensión lexicológica de la traducción, quizás porque ha acaparado excesiva atención, en mi intervención voy a tratar de algunas dificultades para la comprensión del léxico medieval, atendiendo sobre todo a los medios auxiliares para apoyar la competencia de la lengua de la época, especialmente la consulta de los diccionarios y el contexto, así como el examen de los manuscritos, cuando es posible, frente a la recepción acrítica de sus ediciones. Para ilustrar la exposición me serviré de los ejemplos de dos textos en concreto, pertenecientes ambos al período medieval, uno al llamado «francés antiguo» y otro al «francés medio». El primero es el cantar de gesta La Chanson de Roland, cuya elección está motivada, en lo que a la traducción respecta, porque cuenta con buenas ediciones del ms. de Oxford y por la abundancia de versiones modernas que ha conocido ${ }^{5}$. La segunda fuente de ejemplos son los dos textos franceses de la conquista de Canarias, el libro llamado Le Canarien, del siglo XV, perteneciente, por tanto, al período llamado «francés medio»; en este caso la elección ha estado motivada porque, al tratarse del primer documento de la historiografía de Canarias y el primero de la expansión atlántica francesa, también ha conocido numerosas ediciones y algunas traducciones, $y$, sobre todo, porque recientemente un grupo de profesores de la Universidad de La Laguna, entre los que me cuento, hemos publicado una reproducción facsímil de los dos manuscritos $^{6}$, lo que me ha permitido acceder directamente a ellos para llevar a cabo su transcripción y traducción, y a continuación hemos preparado una nueva edición, para la que hube de confrontar mi lectura con la de editores precedentes.

\footnotetext{
4 P.-Y. BAdel (1969), Introduction à la vie littéraire du Moyen Âge, París, Bordas-Mouton, p. 225.

5 He utilizado las de Joseph Bédier y Gérard Moignet en francés moderno, y las españolas de Ángel Crespo, Benjamín Jarnés, Ricardo Redoli, Martín de Riquer y Juan Victorio, todas fácilmente localizables, por lo que me permito omitir los datos bibliográficos, para no alargar innecesariamente este texto. Las referencias a ellas figuran con las letras iniciales del nombre y apellido del traductor.

${ }^{6}$ B. Pico, E. Aznar, D. Corbella, Le Canarien. Manuscritos, transcripción y traducción, La Laguna, Instituto de Estudios Canarios, 2003.
} 
Quienes trabajan sobre textos del pasado saben de las dificultades que plantea la traducción de términos relativos a los realia, cuyo conocimiento se nos escapa. Los llamados, en terminología de Matoré, mots-témoins - tales como franc, baron, vasselage, honor, dame, cortois... - ya han suscitado abundantes comentarios, por lo que me voy a referir a otro aspecto de menor entidad, pero no por ello despreciable, derivado también de nuestro desconocimiento de las realidades medievales para poder traducirlas: se trata de cómo superar lo que Mounin llama dificultades de orden «civilizacional ${ }^{7}$ » frente a términos que designan «cosas» desconocidas para nosotros. No estamos aquí en el plano del significado, sino en el de las cosas designadas, en el plano extralingüístico, y el problema radica en el desconocimiento del denotatum. Para ilustrarlo me voy a servir de dos ejemplos en los que los términos se encuentran en enumeraciones, en las que los lexemas tienen un referente concreto y denotan realidades materiales.

La enumeración, como es sabido, es un procedimiento retórico muy frecuente en la lengua medieval, mediante el cual, por acumulación de referentes se produce un efecto de intensificación cuantitativa. Por tratarse de términos de la misma clase semántica, todos tienen en común los mismos semas genéricos, son elementos de una taxonomía, esto es, poseen un taxema común, que identificamos sin ninguna dificultad, y se distinguen únicamente por semas específicos, que con frecuencia no podemos establecer tan fácilmente.

Así ocurre con los elementos de la enumeración de las armas lanzadas contra los cristianos por los sarracenos, cuyo taxema es 'armas arrojadizas':

Ils lor lancent e lances e espiez

E wigres e darz e museras e agiez e gieser (vv. 2074-2075).

En este caso nuestro conocimiento del referente se reduce a tres elementos, lances, espiez, y darz, y de los otros cuatro, wigres, museras, agiez y gieser, sólo conocemos el taxema 'armas arrojadizas'. En el momento de hacer una traducción, una decisión, muy válida a mi entender, es la que han adoptado tanto Martín de Riquer como los demás traductores, consistente en efectuar una enumeración de armas arrojadizas en español, se correspondan o no; valgan estas dos de 7 y 6 elementos:

Les arrojan lanzas, azconas, venablos, dardos, azagayas, jabalinas y tragacetes (MR).

Les arrojan sus lanzas, astas, picas, venablos, azagayas, jabalinas... (RR).

\footnotetext{
7 G. Mounin (1971), Les problèmes théoriques de la traduction, Cap. XIV : «La philologie est une traduction», París, Gallimard, y (1978), «La traduction», en Grand Larousse de la Langue Française, París, Larousse, VII, p. 6170.
} 
Algo distinto sucede con el término esterminal, que se encuentra entre las piedras preciosas (amatistas, topacios, carbunclos) que adornan el portentoso escudo del pagano Abismo:

Pierres i ad, ametistes e topazes,

esterminals e carbuncles ki ardent (vv. 1500-1501).

Este término, esterminal, es un hápax, registrado como tal en todos los diccionarios $^{8}$, que recogen esta ocurrencia y únicamente informan del taxema 'piedra preciosa'. Aunque A. Ruggeri lo haya puesto en relación con «Esterminans», uno de los nombres bíblicos del diablo y podamos llegar a deducir su connotación por el nombre de su propietario, Abismo, y por su coordinación con carbunclo, seguimos ignorando qué denota. Aquí unos traductores han optado por no traducirlo dejando puntos suspensivos (JB, MR) u omitiéndolo $(\mathrm{BJ})$, o bien por incluir otras piedras preciosas conocidas, sean esmeraldas (AC), porfirio rojo (JV) o rubíes (RR).

También plantean problemas similares las expresiones relativas a acciones insertas en un contexto cultural lejano. Es lo que ocurre con la expresión referida a un momento del combate singular a caballo, la tan debatida pleine sa hanste. El ataque con lanza es un motivo literario fuertemente estereotipado, estudiado hace tiempo por Jean Rychner 9, que establece siete elementos en él: espolear al caballo, blandir la lanza, golpear al adversario, romperle el escudo, destrozarle la loriga, atravesarle el cuerpo y, finalmente, derribarlo del caballo, elemento este al que corresponde la expresión pleine sa hanste (otra variante en los textos es pleine sa lance, Raoul de Cambrai, v. 2756), para indicar el gesto mediante el cual el atacante derriba el cuerpo desarzonándolo, una vez que ha extraído de él la lanza sacudiéndola. El motivo del ataque a la lanza, con parte de sus elementos, se encuentra desarrollado ocho veces en el cantar, del que tomamos un ejemplo en el que sólo falta el elemento «blandir la lanza»:

Sun cheval brochet des esperuns d'or fin, par grant vertut si l'est alet ferir.

L'escut li freinst, l'osberc li descumfist, sun grant espiet par mi le cors li mist,

\footnotetext{
$8 \mathrm{Al}$ aludir a los diccionarios de francés medieval me refiero concretamente a los manuales de A.J. Greimas, Dictionnaire de l'ancien français, París, Larousse, $1^{\text {a }}$ ed. 1968, y A.J. GreIMAS \& T.M. KEANE, Dictionnaire du moyen français. La Renaissance, París, Larousse, $1^{\text {a }}$ ed. 1992, y a los más amplios de F. GODEFroY, Dictionnaire de l'ancienne langue française et de tous ses dialectes du IXe au XVe siècle, 10 vols, Nendeln/Liechstenstein, Kraus Reprint, 1969 (1ª ed. 1881-1902), y de A. TOBLER \& A. Lommatzsch, Alffranæösisches Wörterbuch, Wiesbaden, Steiner (publicado a partir de 1915; actualmente en edición digitalizada).

9 J. RyChNer (1955), La chanson de geste. Essai sur l'art épique des jongleurs, Ginebra, Droz, p. 139 y ss. Remitimos también a $\mathrm{M}^{\mathrm{a}}$ A. ARAGÓN y J.M. FERNÁNDEZ CARDO, El estilo formulario en la épica y en la novela francesas del siglo XIII, Universidad de Oviedo, 1985.
} 
empeint le ben, que mort le fait brandir,

pleine sa hanste l'abat mort el chemin (vv. 1245-1250).

[Pica su caballo con las espuelas de oro fino, y con gran ímpetu fue a atacarlo. Le rompió el escudo, le destrozó la loriga y le metió por el medio del cuerpo su gran azcona; la hunde bien, de modo que se la saca muerto, y con el asta de plano lo derriba en el camino].

En este caso la dificultad de conocimiento no radica tanto en los términos cuanto en la interpretación del gesto, que ha suscitado una serie de artículos, y para pleine sa banste corresponden distintas traducciones: en francés «à pleine hampe» (JB), «de toute la longueur de sa lance» (GM), y en español: «sacudiendo el asta» (JV), «empujando con el asta» (BJ), «a lanza tendida» o simplemente «con el asta» (AC), «lo empala» (RR), y «con el asta de plano» (MR). Esta última interpretación, elegida por Martín de Riquer para dar cuenta del gesto, me parece clara y, a mi juicio no sólo coherente con el desarrollo de la secuencia del motivo, sino también apoyada por sintaxis y fonética, dado que es un complemento circunstancial de modo heredero del ablativo absoluto latino y porque la colisión homonímica entre plena y plana, que se produjo hacia finales del siglo XI, fue responsable de varias confusiones, sin que sea siempre posible establecer la filiación con uno u otro étimo, como señalan los diccionarios históricos ${ }^{10}$; entre las expresiones registradas destaca de plein fouet (Littré), en la que plein indica el movimiento 'en horizontal', por lo que parece válido decidir la equivalencia entre pleine sa hanste y «con el asta de plano» para indicar el golpe horizontal de lanza mediante el que el atacante derriba a tierra a su oponente ya muerto, una vez extraída del cuerpo.

\section{ITERACIÓN SINONÍMICA}

Pasando a otro aspecto de la lengua literaria medieval, voy a detenerme en uno de los rasgos más característicos de la forma-verso, la iteración sinonímica, fenómeno que se sitúa en el marco más amplio de la poética medieval, basada en estereotipos, como muy bien ha puesto de manifiesto Paul Zumthor en su ya clásico ensayo sobre la poética medieval ${ }^{11}$. Los análisis

${ }^{10}$ Con todo, se establece la filiación respecto a planus en varios sintagmas que incluyen plein: Le Robert. Dictionnaire historique de la langue française registra s.v. plein el sintagma «draps pleins», 'unis', la expresión «de plein fouet», en la que plein significa 'à l'horizontale', o «de plein vol». A estas expresiones el Trésor de la langue française añade «armes pleines» o "pleines armes», "pleine terre» (que en francés antiguo designaba un terreno descubierto), así como el término de marina «le plein», del que deriva la expresión «battre son plein».

11 P. ZumThor (1972), Essai de poétique médiévale, París, Seuil, pp. 118-119. 
cuidadosos han puesto de manifiesto que muchas palabras, consideradas a priori sinónimos, no lo eran realmente, lo que voy a ilustrar con varios ejemplos de aparente iteración sinonímica cuya comprensión nos puede orientar en otras tantas opciones de traducción.

En primer lugar, la asociación de un término de semantismo difuso o amplio con otro de significado más preciso que desempeña la función de vector semántico, orientando y restringiendo su significado al aportarle su rasgo específico. Me sirvo de un ejemplo ya utilizado por Claude Buridant ${ }^{12}$, que examino en el texto del cantar, la asociación de los adjetivos fier y orgueilleux. El contenido de fier aplicado a personas no está muy definido, aparte del rasgo de carácter del que tiene un elevado concepto de su propio valor, $y$, como indica J. Batany, «il est tantôt péjoratif (comme le contraire de doux et humble), tantôt laudatif (comme le contraire de coart) $\rangle^{13}$. En el cantar, fier empleado solo tiene un contenido netamente positivo, entrando en las descripciones elogiosas de Carlos o Roldán, o incluso de un noble enemigo:

Carlos "gent ad le cors e le cuntenant fier" (v. 118), "mult par out fier lu vis" (v. 142), Roldán es reconocido "al fier visage e al cors qu'il out gent" (v. 1597), y de un emir se destaca que "cors ad mult gent e le vis fier e cler" (v. 895).

[tiene el cuerpo gallardo y el semblante altivo (v. 118), tenía el rostro muy altivo (v.142); por su rostro altivo y por tener el cuerpo muy gallardo (v. 1597); tiene el cuerpo muy gallardo y el semblante altivo y claro (v. 895)].

Orgueilleux, por el contrario, está claramente connotado como algo negativo, un rasgo de carácter detestable, la soberbia, que debe ser perseguido o aplastado:

Li quens Rollant unkes n'amat cuard, ne orguillos, ne malvais hume de male part, ne chevaler se il ne fust bon vassal (vv. 2134-2136).

Nunca el conde Roldán apreció al cobarde, ni al orgulloso, ni al malvado de baja condición, ni al caballero que no sea buen guerrero].

En el elogio fúnebre a su compañero Oliveros, dice Roldán:

Pur orgoillos veintre e esmaier...

En nule terre n'ad meillor chevaler (vv. 2211, 2213).

No existe en parte alguna mejor caballero para vencer y abatir a los

12 C. Buridant (1980), «Les binômes synonymiques. Esquisse d'une histoire des couples de synonymes du Moyen Âage au XVIIe siècle», Bulletin du Centre d'Analyse du Discours, 4, pp. 5-79.

13 J. Batany(1972), Français médiéval, París, Bordas, p. 85. 
orgullosos].

De ahí que en la asociación de fier con orgueilleux el segundo adjetivo transmite al primero su rasgo peyorativo, de modo que la nobleza, el valor o la intrepidez de las ocurrencias positivas pasan a quedar connotados negativamente como exceso, presunción o arrogancia, y, así el binomio orgueilleux et fier ya sólo puede aplicarse a los enemigos (vv. 28, 2550, 3175).

Del mismo modo en que la acumulación de sustantivos en las enumeraciones produce un efecto de intensificación cuantitativa, la acumulación de adjetivos en este procedimiento retórico de la iteración sinonímica tiene con frecuencia el objeto de crear un superlativo, de manera que las asociaciones tan frecuentes de adjetivos para subrayar la intensidad de las emociones, tales como joianz et liez, bauz et liez, joianz et esbaudis para la alegría, o dolent et triste, dolent et pensif, son perfectamente equivalentes a un superlativo o al adjetivo precedido de adverbio, 'muy alegre' o 'muy triste'. Con esta función de intensificación equivalente a un adverbio entiendo el empleo de merveillus, que califica a todo lo asombroso o extraordinario, que se sale de lo común, en coordinación con otros adjetivos, como se encuentra referido a la batalla:

La bataille est merveilluse e cumune (v. 1320)

La bataille est merveilluse e pesant (v. 1412)

La bataille est merveilluse e hastive (v. 1610)

La bataille est merveillose e grant (v. 1620)

O a las terribles noticias que Carlos tendrá que dar en Aix cuando le pregunten por Roldán:

Jes lur dirrai, merveilluses e pesmes (v. 2919).

Entendidas así muchas de las iteraciones sinonímicas de adjetivos, desde la opción de una traducción «modernizada», se podrían eliminar muy frecuentemente las reiteraciones reemplazándolas por un superlativo o por un intensificador.

\section{CIRCULARIDAD TEXTO-DICCIONARIO Y CONTEXTO}

No obstante, quiero detenerme brevemente para advertir del peligro de ver sinónimos por todas partes y acercarse al texto medieval con ideas preconcebidas. Y aquí quiero mencionar un riesgo que se produce si se recurre únicamente a la información lexicográfica. Los diccionarios encierran, sin duda, tesoros de información y nunca se podría ensalzar bastante su utilidad, pero 
también tienen un lado oculto, lo que podríamos llamar «circularidad textodiccionario». Esto es: para establecer el sentido de las palabras y traducirlas por su equivalente moderno, los autores de los diccionarios (al igual que los autores de las gramáticas canónicas) utilizan la documentación proporcionada por los textos, que autorizan una determinada acepción. Pero cuando se trata de un empleo poco frecuente o inusitado de una palabra que se encuentra coordinada o en una secuencia de varios términos, el diccionario, en este caso, autoriza la nueva acepción a partir de la palabra más conocida y los convierte en puros sinónimos. Resulta así que, cuando se intenta precisar el valor semántico de la palabra en el texto y se recurre al diccionario, se encuentra en él exactamente la misma ocurrencia del texto. $\mathrm{Y}$, con respecto a los empleos inusuales vale la pena retener la afirmación de Buridant de que «dans la rhétorique de la copia, propre au Moyen Âge, le binôme synonymique n'est qu'un des modes de la glose explicative, du commentaire. Il s'intègre dans une architectonique mentale où la nouveauté exige le recours à l'autorité»14; estaríamos, pues, ante lo que Jakobson denomina «traducción intralingual» o «reformulación», consistente en la interpretación de los signos lingüísticos por medio de otros signos de la misma lengua, más o menos sinónimos.

Veamos algún ejemplo de esa circularidad en el Cantar: cuando Roldán, contemplando el rostro de Oliveros herido mortalmente, constata que

Teint fut e pers, desculuret e pale (v. 1979).

Aparentemente, estamos ante una secuencia cuádruple de sinónimos, todos miembros de un mismo paradigma, donde pale sería el archilexema de la serie. Si acudimos a los diccionarios encontramos que precisamente este verso autoriza el significado 'pâle' para teint y para pers, es decir, que ambos indicarían 'pérdida de color' del rostro; y es más, un lexicógrafo como Matoré se apoya también en este verso para poner de relieve que, por causas desconocidas, los términos de color, y concretamente pers, reflejan una curiosa ambigüedad y asumen distintos significados, $\mathrm{y}$, así, junto a las designaciones de tonos de azul, también significa 'pálido'. La acumulación de los cuatro adjetivos, según hemos visto en otras secuencias, tendría también aquí el objeto de poner de relieve la intensidad de la palidez. A esta idea responden sin duda las versiones de este verso tales como «il le voit terni, blêmi, tout pâle, décoloré» (JB), o «marchita está, descolorida y pálida» (AC). Sin desautorizar en modo alguno estas traducciones, quiero precisar, no obstante, mi interpretación de que no estamos ante una secuencia cuádruple se sinónimos, sino ante dos parejas de parasinónimos. Apoyo esta interpretación poniendo en relación esta ocurrencia con dos clichés de extraordinaria frecuencia en los textos medievales: muer la color (o le sen) y perdre la color (o le sen) o, absolutamente, muer et palir (muoit et palisoit, en Tristán). Si a ello se une, de una parte, que teint no tiene en su

${ }^{14}$ Artículo citado, p. 52. 
semema el sema inherente 'ausencia' o 'pérdida' de color, sino, por el contrario, 'presencia', y que también son frecuentes las asociaciones con noir y los contextos en que indica una coloración violenta del rostro producida por la ira, la irritación o la vergüenza, y si tenemos en cuenta, por otra parte, que pers se refiere en los demás contextos, en los que no es coocurrente con pale, al color azulado, interpreto la asociación teint e pers como correspondiente al cliché muer la color, indicando el cambio de color, y la asociación desculuret e pale, en la que los dos adjetivos poseen el sema 'pérdida' de color, como correspondiente al cliché perdre la color.

Un nuevo ejemplo puede servir para poner énfasis en una actitud de un acercamiento al texto medieval libre de ideas preconcebidas en cuanto a ver sinónimos por todas partes y en limitarse únicamente a la información lexicográfica. Cierto es que la asociación de adjetivos, sobre todo en el segundo hemistiquio, adquiere pronto un carácter fijo que los convierte en clichés formularios, pero es igualmente cierto que el poeta es libre de escapar a las asociaciones triviales y puede innovar introduciendo asociaciones de términos inusitadas. Así entiendo la coordinación del verso 2484 del poema: «Noz chevalz sont las e ennuiez». Sin embargo, considerando que ennuié tiene siempre el clasema 'persona', los diccionarios se apoyan precisamente en esta ocurrencia para autorizar la acepción de 'las', cansado, para ennuié, sin duda a partir de la idea preconcebida de la iteración sinonímica bidireccional o reversible. En mi interpretación de que ennuié no ha salido del paradigma de los adjetivos que indican sentimiento pesa el contexto cultural, atendiendo a la consideración especial de que es objeto el caballo sobre los demás animales — tiene nombre propio, $y$, atendiendo a la lengua, se comprueba que chief, connotado positivamente frente a teste, sólo se aplica a humanos y a corceles_-, lo cual permite fácilmente el cambio clasemático del adjetivo sin alterar sus rasgos de contenido, por lo que no se ha convertido en sinónimo de las. He de decir, no obstante, que las traducciones que se hacen de este verso son: «nos chevaux sont las et recrus» (JB), «nos chevaux sont las et à bout de forces»(GM), «nuestros caballos están cansados y rendidos» (MR, JV), «nuestros caballos están flojos y exhaustos»(RR), y «nuestros caballos están ya muy cansados» (AC), aunque no sé si ha sido por estimar la traducción más adecuada al lector actual.

En mis dos interpretaciones anteriores he acudido a contextos extradiscursivos para poner de manifiesto los límites de la lexicografía, las limitaciones de la traducción a partir únicamente de la información de los diccionarios. Parece ocioso insistir aquí sobre la importancia del contexto, pues, a la hora de comprender un texto entrarán forzosamente en consideración, además del sistema funcional de la lengua al que atiende el diccionario, los semas aferentes del contexto lingüístico, tanto los situados en el plano sociolectal como en el idiolectal, además del contexto pragmático. 
El contexto intralingüístico nos permite descubrir relaciones de significado distintas a las registradas en la lexicografía. Así, los diccionarios de francés antiguo registran braire como sinónimo de crier, sin hacer mención de la solidaridad léxica de implicación con 'asno', como en francés actual, o con otro animal. En Le Robert. Dictionnaire historique de la langue française leemos s.v. braire: «en ancien et moyen français le mot est synonyme de crier; les deux verbes sont souvent employés en couple ; le sujet désigne une personne ou, plus rarement, un objet». Con estas informaciones, al encontrar en nuestro texto del Cantar braire coordinado con benir, podríamos deducir que braire es un término no marcado con respecto a henir, que sí está marcado por el sema inherente 'caballo'. Pero la situación en el contexto discursivo nos precisa su relación: es el momento en que se reúnen las tropas sarracenas del emir Baligán antes de la última gran batalla, $\mathrm{y}$

Cil d'Occiant i braient e henissent

Arguille si cume chien glatissent (vv. 3525-3526)

Entendemos que la coordinación con benir autoriza el cambio de semas macrogenéricos del sujeto y que cil d'Occiant reciben el rasgo aferente 'animal', virtualizándose el rasgo 'humano', asociación implícita con desplazamiento de semas que aparece ya de forma manifiesta en el verso siguiente, si cume chien glatissent, apoyando esta interpretación. Parece, pues, adecuado traducir el primer verso atendiendo al contexto situacional inmediato y no al diccionario, y, ya que los verbos henir y glatir indican comportamientos animales, es oportuno interpretar del mismo modo braire, y no con el significado de 'chillar', 'vocear' que se encuentra en las traducciones utilizadas, salvo en una:

Y los de Occián aúllan y relinchan, y ladran los de Argulla como perros (RR).

El texto de Le Canarien nos va a permitir a continuación ilustrar otras dificultades de comprensión de la lengua medieval que también necesitan «linternas». Dado que fuera del ámbito de los historiadores o de los eruditos canarios este texto no es conocido, he de aclarar brevemente que la crónica redactada por los clérigos de la expedición se conserva sólo en dos copias manuscritas diferentes del siglo $\mathrm{XV}$, que convencionalmente se denominan «G» $\mathrm{y}$ «B», por las iniciales de Gadifer y Béthencourt, respectivamente. El primero de los manuscritos fue realizado sin duda en un buen taller de copistas profesionales en cuidada caligrafía gótica libraria antes de 1420, probablemente bajo la supervisión de Gadifer de La Salle, y el segundo, la copia «B», es un manuscrito familiar redactado hacia 1488, escrito en letra bastarda cursiva, a instancias o bajo la dirección de Jean $\mathrm{V}$ de Béthencourt. De ambos existen 
numerosas ediciones y algunas traducciones ${ }^{15}$, y, como ya dije, he trabajado sobre ellos para efectuar su transcripción, traducción y edición.

\section{4. «FALSOS AMIGOS»}

Casi resulta ofensivo mencionar aquí los que, utilizando un galicismo, se denominan «falsos amigos», las palabras de distintas lenguas que coinciden en la forma pero difieren en el significado. Este error, que parece burdo y sólo propio de principiantes, se produce, no obstante, con cierta frecuencia cuando los significantes de una lengua van unidos en diacronía a diferentes significados, por lo que, manteniéndose la forma, el contenido ha variado, e incluso un traductor avezado puede cometer distracciones. Sin pretender en modo alguno menoscabar la imagen de traductores respetables, sí me detengo en mostrar la insidia de algunos de estos falsos amigos.

En el ms. B, fol. 72v, leemos precediendo a una ilustración, como título de capítulo escrito con tinta roja, Comment ledit seigneur a tous ces amis du païs, pour leur dire adieu, leur donna a diner et les festia, que, sin excesiva atención, es traducido por «Cómo el dicho señor invitó a cenar y a festejar a todos sus amigos del país, para despedirse de ellos» (AC), cuando es conocido que disner - como desjeuner-, en francés antiguo y medio, conserva todavía su relación con el étimo disjejunare, 'romper el ayuno', y se refiere a la primera comida principal del día, cuya hora era variable, entre tercia y sexta generalmente, para el que se levantaba bastante antes del amanecer; en el francés de Québec se mantiene dîner para lo que en Francia es déjeuner, la comida de mediodía. Parece claro que lo que el señor de Béthencourt ofreció a sus amigos fue un almuerzo, no una cena.

En otros casos el falso amigo es más taimado, y, junto al significado habitual, puede tener un referente específico no usual. En mi opinión, es lo que ocurre con la descripción que hacen los cronistas de las islas de Tenerife y Gran Canaria:

L'isle d'Enfer, qui se dit Tenerefix, est en maniere d'une herse, pres

\footnotetext{
15 No sería oportuno incluir aquí un largo apartado de referencias bibliográficas, por lo que remitimos a la introducción de Le Canarien. Manuscritos, transcripción y traducción, citado, y a B. PiCO y D. CORBELLA, «La transmisión de Le Canarien», en Homenaje al Profesor Don Francisco Javier Hernándę, Valladolid, Departamento de Filología Francesa y Alemana. Universidad de Valladolid-APFUE, 2005, pp. 455-473, donde se describen las principales ediciones y versiones. Para el objeto de esta conferencia puede bastar con la simple enumeración de los editores a los que aludimos. Las ediciones de «B» que citamos son la de Pierre BERGERON (1630), Édouard Charton (1855), Gabriel Gravier (1874), Richard Henry Major (1877); de «G» la de Pierre MARgry (1896); y de ambos manuscritos, Elías Serra y Alejandro Cioranescu (1959, 1960, 1964) o solo traducciones por A. CIORANESCU (1980 y nueva edición 2005).
} 
que ainsi que La Grant Canare, La Grant Canare ... est en maniere de herce $(\mathrm{G}, 34$. B 48, 48v).

[La isla del Infierno, llamada Tenerife, tiene forma de gran candelabro, casi como La Gran Canaria, La Gran Canaria ... tiene forma de gran candelabro]. Hasta ahora se había venido traduciendo que la forma de las islas era como un «rastrillo», cuando se puede comprobar que, junto a esta acepción usual, rastrillo o reja, desde finales del siglo XII coexiste otra específica que designa un gran candelabro de iglesia erizado de puntas en las que se clavan los cirios, o «tenebrario», candelabro triangular de quince velas utilizado en los oficios de tinieblas; teniendo en cuenta que los redactores eran clérigos, uno de ellos procedente de una abadía benedictina, tenían que estar familiarizados con esta acepción, que, a mi juicio, se adecua mejor a la imagen que tendrían de la orografía de las islas los exploradores que las bordearan desde el mar.

Es similar lo que sucede con un pasaje del ms. B que relata las gestiones de Jean de Béthencourt para obtener una copia de las escrituras por las que el rey castellano Enrique III le otorgaba el señorío de las islas conquistadas, con el derecho de acuñar moneda y de percibir el quinto real de las mercancías. El texto dice que el noble normando

desiroit avoir ces lestres toutes faites, grossees et scellees $(B, 52 \mathrm{v})$.

[quería obtener una copia completa, legalizada y sellada de las escrituras].

En las distintas ediciones grossees pasa a transcribirse como grosses (Bergeron, Major) o grossies (Gravier, Serra-Cioranescu) y a traducirse como «engrossed» en inglés (Major), «extendidas» en español (Cioranescu), y sólo en la versión de Charton se encuentra correctamente actualizado el participio como «grossoyées». Aquí se trata de la acepción jurídica que adquiere el verbo grosser desde el siglo XIV, y que alude al tipo de letra de las escrituras notariales, en las que se distinguía, por una parte, la minuta, esto es el original de una escritura notarial en letra «menuda», de la que el depositario no puede desprenderse y de la que se hacen las copias, y, por otra parte, la grossee, la copia legalizada escrita en caracteres más gruesos que los de la minuta y revestida de la fórmula ejecutoria, acepción jurídica que deriva del latín medieval grossa, que se utilizó desde la expedición de actas del papa Inocencio III redactadas en letras gruesas.

También puede suceder que la causa de una traducción poco fiel resida en una homonimia que se le escapa al traductor. Así, en la instrucción o catecismo que redactan los clérigos para adoctrinar a los canarios, se habla de la construcción del arca de Noé y se cuenta que el arca debía ser calafateada por dentro y por fuera con betún de Judea, sobre lo que volveremos más adelante, y que el betún de Judea es una cola tan fuerte que las maderas unidas con ella no podría ser separadas si no es con sangre de fleur de femme, que ha sido 
traducido como «flor de mujer», cuando tal expresión no tiene referente en español. En este caso, no sé si se ha optado por un eufemismo pudoroso, puesto que fleur, deverbal de fluer, se refiere al flujo menstrual: la forma flueur del francés antiguo había llegado en francés medio a la homonimia con fleur, tal vez por influencia metafórica, de modo que una acepción de fleur es la menstruación(en su anatomía, Ambroise Paré señala que «les femmes appellent leur flux de sang par la matrice ... fleurs rouges»).

\section{GRAFÍA Y PUNTUACIÓN}

Dejo para el último apartado el recurso que, a mi parecer, puede arrojar más luz sobre los pasajes poco claros de un texto: la consulta directa de los manuscritos, cuyo acceso es, en ocasiones, algo más dificultoso que el de las ediciones impresas, pero, generalmente, no imposible en la actualidad. Si se trata de hacer una edición, tal consulta directa es, ni que decir tiene, inexcusable; y, en lo que a mi limitada experiencia concierne, ha sido el único medio válido para apoyar una nueva lectura al poner en cuestión la validez de lecturas anteriores no convincentes. Naturalmente, en este caso la nueva lectura viene respaldada por la familiaridad con la lengua medieval y por la habituación a la grafía de los manuscritos.

Es sabido que la grafía de las letras góticas a trazos puede provocar dificultades de lectura y, consiguientemente, discrepancias de interpretación, como voy a ilustrar mediante algunos ejemplos. En los dos primeros, la confusión se debe a la coincidencia de grafía para los valores de $u$ vocal y $v$ consonante: en medio de palabra, la única grafía para ambos fonemas es $u$, y en posición inicial la grafía $v$ - tiene los dos valores.

En varias ocasiones figura en los textos una frase de la que forma parte la palabra que nosotros leímos sin dificultad convine — savoit nostre convine-, que, para mejor compresión, sitúo en un breve contexto:

Alfonce, lequel Bettencourt avoit amené pour estre truchement, comme dessus est dit, lequel demouroit continuelment avecques nous et savoit nostre convine et nostre povreté, et tiroit du tout a nostre destruction (G 13v),

[habla del trujamán Alfonso, que, "por estar continuamente con nosotros, conocía nuestra situación y nuestra escasez, y lo único que pretendía era nuestra muerte"].

Et vint Pietre le Canare parler a eulx et le filx du roy qui s'appelle Artamy, et des autres Canarez grant planté ... mais quant ilz virent 
nostre convine et que nous estoions po de gens, en la fin ilz nous cuiderent traïr (G 32, B 44),

[fueron a hablar con ellos Pedro el Canario y el hijo del rey llamado Artamy, y gran número de canarios, pero cuando vieron nuestra situación y que éramos pocos, finalmente pensaron en traicionarnos]

Et Gadifer y a esté par deulx saisons tout a effect pour voir leur maniere \& les gouvernement, leur estat \& leur convine [de los habitantes de Gran Canaria] (G, 34v).

Los editores precedentes efectuaron otra lectura: Margry decidió transcribir coutume, que es descartable, mientras los demás se inclinaron por comune (Bergeron, Major, Gravier, Serra-Cioranescu), lo que hace poco comprensible la frase, en tanto que el término convine, que figura como variante de la entrada covine en los diccionarios de Godefroy y Tobler-Lommatzsch, se refiere a la situación en la que se encuentran personas o cosas; por otra parte, convine precedido de posesivo como complemento de verbos de conocimiento es de uso frecuente en las crónicas, por lo que creemos que esta es la lectura adecuada.

La grafía también está en el origen de otras lecturas que consideramos erróneas. El traidor Bertin ha invitado a cenar a un grupo de canarios, a los que piensa capturar y vender como esclavos, y, mientras sus huéspedes están durmiendo,

quant Bertin vist que temps fu, il se mist devant leur uis l'espee en la maint toute nue (G9).

[Cuando Bertin vio que era el momento, se plantó delante de su puerta blandiendo la espada].

Para nosotros es evidente que se colocó delante de su puerta, uis, y no delante de su rostro, vis, como leyó Margry.

A este editor se debe otra lectura inadecuada que luego fue reproducida por los editores sucesivos, sin advertir la contradicción con el contexto:

Et ancrerent en un grant port qui est entre Telde et Agouimes, et la sur le port vint des Canares environ $\mathrm{V}^{\mathrm{C}}$ et parlerent a eulx. Et venoient a la barge dix, XII, tous ensambles noant aprés que Gadifer les eüst asseürez (G 18v).

[fondearon en un gran puerto situado entre Telde y Agüimes, adonde acudieron a hablar con ellos unos quinientos canarios. Después de que Gadifer les garantizara su seguridad, se acercaban nadando al barco en grupos de diez o doce]. 
Pese a que está claramente escrito noant, gerundio del verbo noer, 'nadar', Margry transcribió n'osant, gerundio de oser, 'atreverse', precedido de negación, error que reprodujeron los editores que le sucedieron y que dio lugar a una traducción poco coherente.

No es infrecuente que algunas malas lecturas tengan su origen en el primer editor, que condiciona de tal manera a los que le suceden que el mismo error se reproduce una y otra vez. Veamos algún caso. La primera edición del ms. «B» estuvo a cargo de Bergeron en 1630. En el fragmento que reproducimos, fol. 16v, se relata cómo, tras la partida del traidor Bertin con sus prisioneros en las naves españolas, sus cómplices, abandonados en tierra y temerosos de las represalias de Béthencourt y Gadifer, se apoderan de una barca en la que se dirigen hacia la costa africana, pues las naves españolas ya se encontrarían a medio camino hacia España:

eulx craignant l'ire <et> le couroux d'icellui, comme gens deesperés, prindrent leur chemin a tout le batel droit en terre de Mores, car les naves pouent bien estre my voie de la et d'Espaigne. [desde De Mores, $3^{\mathrm{a}}$ línea izquierda]

Tal vez por distracción, Bergeron escribió «car les Mores» en lugar de «car les naves» y, tras él todos los demás editores (Charton, Major, Gravier, Serra-Cioranescu), que aseguran haber consultado el original; estos últimos, percibiendo la inconsecuencia, indican en nota: «Error grave del copista de B, por cuya presencia se altera totalmente el sentido de la frase, que todas las ediciones anteriores reproducen...», cuando es evidente que, en esta ocasión, el copista no merece que se le impute el error.

Mucho más sorprendente y curiosa, por cuanto ninguno de los editores ha advertido el error, resulta otra lectura de Bergeron. En el pasaje del catecismo para instruir en la religión a los canarios relativo a la construcción del arca de Noé y a calafatearla, al que hemos aludido, dice el texto:

l'oindroit dedens \& dehors de bettuun (bettuun est ung glu si fort et si tenant que quant deulx piesses de fut en sont assemblés et jointes on ne les peult par nul art desassembler, sinon par sanc naturel de fleur de femme, et le treuve l'en flotant es grans lacs de Judee sur les aygues) (B 33-33v).

[la untaría por dentro y por fuera con betún —el betún es una cola tan fuerte y tan adherente que si se juntan y se pegan con ella dos trozos de madera, no existe forma alguna de separarlos, a no ser con sangre natural de menstruación femenina; se encuentra flotando en la superficie de las aguas de los grandes lagos de Judea]. 
Es muy extraño que Iudee haya sido transcrito por Bergeron como Indie, India, y nadie haya reparado en su mala lectura, cuando el betún de Judea, residuo del petroleo, era conocido desde la antigüedad por los pueblos de Oriente, que lo utilizaban en pintura y para impermeabilizar; los antiguos egipcios se sirvieron de él para embalsamar las momias, y en Babilonia era usado como mortero y para calafatear embarcaciones, siendo los yacimientos de betún más célebres los del valle del Jordán, el famoso «betún de Judea».

Para terminar, vamos a ver cómo en la transmisión de un texto a las dificultades de la lectura se añade el establecimiento de los signos de puntuación por parte de los editores, que pueden alterar sensiblemente la comprensión. Cuando los cronistas están describiendo en el ms. «G» a las mujeres de Fuerteventura, su atuendo y la forma de llevar el cabello, dicen:

\& portant grans cheveulx \& crespez, qui sont taillé devant le front en la maniere que les hommes, les portant en buytefale; elles alectent leur enfens a la memmelle ( $\mathrm{G} 36)$.

[tienen el cabello largo y rizado, cortado sobre la frente como lo llevan los hombres, todo alborotado].

La dificultad del término buytefale ha llevado a interpretarlo como un topónimo y a puntuar así: «grans cheveulx \& crespez, qui sont taillé devant le front en la maniere que les hommes les portant. En Huytefale elles alectent leur enfens a la memmelle», y a aclarar en nota que Huytefale sería «un nombre de lugar muy estropeado por el copista, en donde los hombres llevarían cierto corte de pelo, o donde las mujeres amamantan al pecho jcosa no bastante notable! Proponemos la corrección, que llevamos a la traducción: En ceste ysle». La puntuación que nosotros hemos establecido no violenta la sintaxis, funcionando en buytefale como complemento de modo; acudiendo al diccionario de Tobler-Lommatzsch encontramos la entrada buitefale, de traducción poco clara («Kirmes?»), y diversas variantes, entre ellas hurebale, que registra Godefroy e interpreta como 'jolgorio'. Nos parece posible que los cronistas, al señalar que las mujeres llevaban el pelo rizado cortado sobre la frente como los hombres, en buytefale, pretendieran indicar, de modo gráfico y expresivo, que lo llevaban en desorden y muy alborotado.

Otro ejemplo curioso de cambio de sentido propiciado por lectura y signos de puntuación es el que vamos a ver como final de esta intervención. Cuando Béthencourt regresó a Canarias precedente de España, habiendo obtenido sólo para sí el señorío de las Islas, con total olvido de su compañero que había llevado todo el peso de la conquista y padecido innumerables penalidades, las relaciones entre ambos capitanes se deterioraron y llegaron a ser de verdadera hostilidad, de modo que Béthencourt comienza a fortificarse, 
y lo mismo hace Gadifer por su parte,

et rescrirent l'un a l'autre ; et y avoit aux lettres que messire Gadifer rescrip a monseigneur de Bethencourt seullement pour toute escriture : «Se vous y venés, se vous y venés, se voous y venés...», et non autre chose. Et adonc monseigneur de Bethencourt lui rescript par son poursuivant nommé : «Se je puis, se vous y trouvés, se vous y trouvés, se vous y trouvés... » (B 43v). [se intercambiaron cartas, y en la carta que escribió el señor Gadifer a monseñor de Béthencourt lo único que había escrito era «Como vengáis aquí, como vengáis aquí, como vengaáis aquí... », y nada más. Entonces el señor de Béthencourt mandó con uno, al que nombró su heraldo de armas, esta respuesta: «Como pueda y estéis ahí, como estéis ahí, como estéis ahí... »].

Ésta es mi propuesta, apoyándome especialmente en el indicio del calderón rojo que precede a la respuesta de Béthencourt, que marca generalmente una pausa equivalente al punto o, en este caso, el paso al estilo directo en la narración, con lo que la frase adquiere sentido. Sin embargo, algunos de los editores anteriores, quizás por dificultades de comprensión (Bergeron, Charton), omitieron el pasaje, en tanto que otros (Major, Gravier, Serra-Cioranescu), sin conceder importancia al signo, interpretaron Sejepuis en una sola palabra como nombre propio, leyendo «son poursuivant nommé Sejepuis : - Se vous y trouvés, se vous y trouvés... », a la vez que explican en nota que «el nombre Sejepuis, que significa 'si yo puedo', es bastante extraño; pero era regla de los poursuivants, especie de escuderos al mismo tiempo que estafeta, heraldo y rey de armas, adoptar nombres curiosísimos». Puedo estar equivocada, pero para mí lo curiosísimo es que al establecer una edición se pueda llegar a un sinsentido por prescindir de las indicaciones del manuscrito.

No es mi intención, de ningún modo, atacar o infravalorar el trabajo de los editores precedentes, quienes, con su labor paciente y meritoria, pusieron a nuestro alcance obras del pasado y aseguraron su difusión, pero sí he pretendido poner de relieve que aquí, como en cualquier campo científico, las comparaciones no son odiosas, muy al contrario: se avanza gracias a que se confrontan unas y otras interpretaciones, se pone en cuestión lo recibido y no se acepta dogmáticamente nada como definitivo, y, naturalmente, menos aún, la producción propia ${ }^{16}$. No sé si las propuestas que acabo de hacer alumbran

\footnotetext{
$16 \mathrm{Al}$ respecto es extraordinariamente ilustrativa y merecedora de los mayores elogios la actitud de honestidad intelectual de Jean RYCHNER, cuya puntuación de su edición de los Lais de Marie de France fue corregida por nuestro colega Manuel BRUÑA en su tesis doctoral. El prestigioso editor dice así al comienzo de un artículo: «Je suis, hélas! on ne peut plus d'accord avec les critiques que Manuel Bruña Cuevas fait à la ponctuation de mon édition des Lais relativement au discours indirect libre. Le rouge me monte au front à me relire, mais ma honte a cela de bon,
} 
ENTREC ULTURAS Número 1. ISSN: 1989-5097. Fecha de public ación: 27-03-2009

algo el largo camino de la traducción del francés medieval, pero sí sé que otros llegarán con mejores linternas para iluminarlo más.

pour moi en tout cas, qu'elle renouvelle mon besoin de voir plus clair... » («Le discours subjectif dans les Lais de Marie de France. À propos d'une étude récente», Revue de linguistique romane, 209210,1989 , p. 57). 